\title{
Simulation of water supply networks using modern means of information technology
}

\author{
Jerzy Kotowski ${ }^{1, *}$, Jacek Oko ${ }^{1,2}$, and Monika Żygadło ${ }^{1}$ \\ ${ }^{1}$ WrUST, Faculty of Electronics, Wroclaw, Wybrzeże Wyspiańskiego 27 Str., Poland \\ ${ }^{2}$ Vice-Chancellor for Informatization and the Director of the Centre for Networking and \\ Supercomputing at WrUST
}

\begin{abstract}
We present in turn the development of computer technology from the beginning of its creation on our planet. Then we discuss the development of information technology at our university. At the end, we present a selected technical problem which is the task of simulation of the water supply network. We present mathematical models of these issues and algorithms for their solutions requiring the use of a computer.
\end{abstract}

\section{Introduction}

The presented work can be easily divided into two separate parts. In the first part, we present the development of computer technology on our planet. The history of computer technology begins during World War II. We start from the first, shy steps, focusing, inter alia, on the creation and use of the first computer called ENIAC, whose construction began in 1943. ENIAC helped to win the Second World War. It was used in the Manhattan project to simulate the explosion process in an atomic bomb.

Then we present milestones in computer development that have led to significant changes in the analysis of thinking processes in the human brain. We describe, among other things, the role of the Deep Blue computer ending at the Watson computer.

In this part of the work, we describe the activities carried out at the Wroclaw University of Science and Technology regarding the development of a computer network and the use of computers for the introduction of new education techniques in the Lower Silesia region.

In the second part of the work, we describe the process of determining the flows in a nonlinear, intensive network working for the needs of a large urban agglomeration. Subsequently, we present the model of this task. We transform this model into a static optimization task with a non-linear objective function and linear constraints.

Then, using known methods in the field of optimization techniques, we reduce this problem to unrestricted form. The number of variables in the reduced problem is equal to the number of meshes in the analyzed network, which is in practice much smaller. We solve the problem on the computer using the modified Newton's method.

At the end, as usual, we present a plan for further work.

\footnotetext{
${ }^{*}$ Corresponding author: jerzy.kotowski@pwr.edu.pl
} 


\section{The development of computer technology}

We will start this section with the presentation of a commonly used measure of processor speed, namely a parameter called flops. Short for floating-point operations per second, a common benchmark measurement for rating the speed of microprocessors. Floating-point operations include any operations that involve fractional numbers. Such operations, which take much longer to compute than integer operations, occur often in some applications.

Due to computer development we have to use bigger values. Some of them look as follows : megaflops $-10^{6}$ flops, gigaflops $-10^{9}$ flops, teraflops $-10^{12}$ flops, petaflops $-10^{15}$ flops, eksaflops $-10^{18}$ flops, etc. Note that $10^{15}$ means one million billion! A lot...

\subsection{ENIAC}

Short for Electronic Numerical Integrator and Calculator, the ENIAC was the first electronic computer used for general purposes, such as solving numerical problems. It was invented at the University of Pennsylvania in an effort to calculate artillery firing tables for the United States Army's Ballistic Research Laboratory. The name suggests that the first applications consisted in numerically solving big systems of differential equations.

Its construction began in 1943. Although it was not completed until the end of World War II ENIAC was used for the design of nuclear weapons as a part of the Manhattan Project in Los Alamos Laboratory. ENIAC helped in a significant way to win the Second World War. He was used in the Manhattan project to simulate the explosion process in an atomic bomb.

By 1956, the end of its operation, the ENIAC occupied about 167 square meters and consisted of almost 20,000 electron tubes, 1,500 relays, 10,000 capacitors, and 70,000 resistors. It also used 200 kilowatts of electricity, weighed over 30 tons, and cost about $\$ 487,000$.

\subsection{Stages of computer development on planet Earth}

The table below presents selected milestones in the development of computers.

Table 1. Selected milestones in computer development.

\begin{tabular}{|c|c|c|}
\hline Year & Computer & Speed \\
\hline 1946 & ENIAC & $5000+350 * 40$ per sec. \\
\hline 1976 & Cray-1A & 133 MFLOPS \\
\hline 1983 & Cray X-MP & 235 MFLOPS \\
\hline 2002 & Earth-Simulator & 35.9 TFLOPS \\
\hline 2004 & IBM Blue Gene & 70.7 TFLOPS \\
\hline 2005 & IBM Blue Gene & 140 TFLOPS \\
\hline 2008 & IBM Roadrunner & 1 PFLOPS \\
\hline
\end{tabular}

But this is not all! Deep Blue was a chess-playing computer developed by IBM. It is known for being the first computer chess-playing system to win both a chess game and a chess match against Garry Kasparov, a reigning world champion under regular time controls. 
Deep Blue and Kasparov played each other on two occasions. The first match began on 10 February 1996. Deep Blue lost this match with a score of 4-2. In May 1997 there was a rematch. Deep Blue won game six, therefore winning the six-game rematch $3 \frac{1}{2}-2 \frac{1}{2}$ and becoming the first computer system to defeat a reigning world champion. It forced our civilization to change the definition of intelligence. Until now, it was assumed that intelligence is a feature belonging to a human being and not to any electrical or mechanical device. From the definition understood in such a way, it appeared that the computer would never win in a chess match with a reigning world champion.

\subsection{Watson}

Watson is a supercomputer created by IBM to answer questions asked in natural language. Its name honors the founder of this company, Thomas J. Watson. The supercomputer is being developed as part of the DeepQA research project. It uses a combination of algorithms for natural language processing, information retrieval, knowledge representation, automatic inference and machine learning. It has 2880 cores, $15 \mathrm{~TB}$ of operating memory and does not use an internet connection.

As part of the presentation of its abilities Watson appeared in the TV game show Jeopardy! (Whose Polish equivalent was Va banque) in the three-day game in 2011. Watson took first place with a result of $\$ 77,477$. Its opponents earned respectively $\$ 24,000$ and $\$ 26,000$. Here are some key events that happened along the Watson's way.

- February 2011: Victorious at Jeopardy. Watson competes on Jeopardy and defeats the TV quiz show's two biggest all-time champions.

- January 2014: A business unit is born. IBM launches the IBM Watson Group, a business unit dedicated to developing and commercializing the technology. Later that year, it opens a global headquarters in New York City's "Silicon Alley."

- April 2015: Honing in on health. We each generate one million gigabytes of healthrelated data across our lifetime, IBM says. The Watson Health business unit aims to help patients, physicians, researchers and insurers use that data for better health.

- December 2015: A new IoT branch. IBM opens a new Watson internet of things (IoT) global headquarters in Munich, Germany.

- February 2016: Watson for U.S. president? Though it says it's not affiliated with IBM, the Watson 2016 Foundation launches the Watson for President website to help promote the technology's presidential prospects.

- May 2016: Eight languages and counting Watson begins learning Korean, bringing the total number of its conversational languages to eight. Also part of its repertoire are English, French, Italian, Spanish, Brazilian Portuguese, Japanese, and Arabic.

- May 2016: Off to (cybersecurity) school. IBM Security announces a year-long research project through which it is collaborating with eight universities to help train Watson to tackle cybercrime.

- June 2016: Learning to drive. Local Motors debuts Olli, the first self-driving vehicle to integrate Watson's capabilities. The 12-passenger electric vehicle analyzes and learns from transportation data produced by more than 30 sensors embedded throughout the vehicle. It also leverages four Watson developer APIs to enable smooth interactions between passengers and the vehicle.

Also cancer treatment is now more effective thanks to Watson. Watson has an access to 600,000 scientific papers, 15 million pages of textbooks and 1.5 million medical records from patients around the world. Many hospitals and clinics are currently implementing Watson in Poland. 


\section{Cloud Computing in Central and Eastern Europe}

Changes in education will not be possible without a flexible system that would support cooperation between all types of schools and interactions with businesses. IBM Smarter Cloud Orchestrator (SCO) at Wroclaw University of Technology is a base on which such systems for the entire region of Lower-Silesia will be built. The first phase of the project consists of implementation of an IT Cloud for education at the University with the architecture and technology already in place to support future extension. A private cloud for education, a flexible cloud for the university vital administration systems, and the system built on IBM SCO will become a core engine for Lower Silesian Educational Cloud [1].

\subsection{IBM in Poland}

IBM had subsidiaries and operations in 70 countries in its early years. IBM first appeared in Poland in 1935 as the Polish Hollerith and later in 1938 as Watson Business Machines. IBM returned back to Poland in 1991 and has been operating here since. The Consortium offers a job for thousand people. Also, and quite importantly for the younger generation, it offers positions for candidates who speak English and one or more of the European languages: French, German, Dutch, Italian, Spanish, Portuguese, Norwegian and Russian.

The IBM Delivery Centre Poland in Wroclaw also provides implementation services, project management services, and enterprise IT process management services. It employs specialists in the disciplines presented below, among others: Junior \& Experienced IT Specialist, Project Manager, IT Architect, System Operators, Client Support Manager and Service Coordinator.

\subsection{Wroclaw University of Science Technology and IBM Corporation}

30 September 2009, in the Head Office of Polish Information and Foreign Investment Agency in Warsaw, there was signed a contract between IBM Poland and our government, concerning the location of the new IBM Integrated Delivery Center in Wroclaw. During the signing ceremony it was assumed that at WrUST, a special team would be created aimed at cooperation with IBM. The University started to fulfil its obligations in the field of postgraduate training for employees and education for future IBM staff.

In May 2010, after the creation of the University Competence Center on Cloud and the development of the cooperation of many areas related to Cloud, called Multipurpose Cloud Center (mc2), the Faculty of Electronics and IBM Corporation introduced an innovative internship program for students of PWr. Apart from its educational character in the informatics area and in practical tasks, it offers flexibility as task work can be done from any remote location during all working hours. However, the most important aspect of the program is that it offers the students the opportunity to prove themselves and demonstrate their creativity. IBM appreciates the commitment of WrUST in cooperation. Three academic staffs from WrUST were rewarded by valuable IBM Faculty Award [2].

World of Innovation - this was the title of the conference, which took place on $3^{\text {rd }}$ April 2012, at Wroclaw University of Technology, concerning the latest achievements in the IT field, and was organized in cooperation with IBM Global Services Delivery Centre in Wroclaw.

During the conference, WrUST and IBM Haifa Research Laboratory signed the Declaration of Intent, concerning future cooperation. Collaboration was concerned with creating several laboratory groups for research and development of the latest IT technologies, in the field of Cloud Computing, IT modeling, as well as problems related to Optimization. 


\subsection{IBM Smarter Cloud Orchestrator at WrUST}

Orchestrator is a workflow management solution for the data center. Orchestrator lets to automate the creation, monitoring, and deployment of resources. Cloud orchestration solutions from IBM are designed to reduce the IT management complexities introduced by virtual and cloud environments and accelerate cloud service delivery, allowing enterprises to quickly respond to changing business needs.

IT administrators perform many tasks and procedures to keep the health of their computing environment up-to-date and their business running. Tasks might include the diverse activities. Individual tasks and subtasks are automated, but typically, not for the whole process.

By using Orchestrator, we can carry out the following tasks: Automate processes in the data center, regardless of hardware or platform, automate IT operations and standardize best practices to improve operational efficiency and connect systems from different vendors without having to know how to use scripting and programming languages. Orchestrator provides tools to build, test, debug, deploy, and manage automation in environment. The standard activities defined in every installation of Orchestrator provide a variety of monitors and tasks with which it is possible to integrate a wide range of system processes.

Any IT organization can use Orchestrator to improve efficiency and reduce operational costs to support cross-departmental objectives. Orchestrator provides an environment with shared access to common data. By using Orchestrator, an enterprise can evolve and automate key processes between groups and consolidate repetitive manual tasks. It can automate cross-functional team processes and enforce best practices for incident, change, and service management.

Future education will be not only widely available, but also much smarter than today. Due to a rapid growth of technologies, among which cloud computing plays a key role, the education supporting systems will use all available information in order to personalize the process of teaching. IBM says it is already happening and predicts a fast development of such technologies within 5 years. The revolution of process of teaching will affect all levels of education, not only academia. The adoption of new mentality as well as the development of necessary systems will obviously take some time, however it is particularly academia's role to lead the process.

All the changes in education will not be possible without a flexible system that would support cooperation between all types of schools and interactions with businesses. IBM Smarter Cloud Orchestrator (SCO) at WrUST is a base on which such systems for the entire region of Lower-Silesia will be built. The first phase of the project consists of implementation of an IT Cloud for education at the University with the architecture and technology already prepared to support future extension. The system at the WrUST contains the virtual platform of the system based on four servers IBM Flex System x240. At WrUST System SmartCloud Orchestrator was installed on virtual machines with operational system Red Hat Enterprise Linux 6.3 x64. The components of Cloud System are as follows:

- Smart Cloud Orchestrator,

- IBM Image Construction and Composition Tool,

- IBM Virtual Image Library,

- IBM Process Center,

- IBM Process Portal.

\section{Simulation of flows in the water supply network}

In order to simulate digital flows in a non-linear transport network (gas, water, etc.), instead of solving the system of type I and II nonlinear equations, Kirchhoff's law solves the problem 
of static optimization with a convex target function and linear equality constraints (Kirchhoff's I law).

The optimization problem can be reduced. The idea of reduction consists in unraveling linear constraints and making appropriate substitutions in the objective function. The number of variables in the reduced problem is equal to the number of meshes in the network (circular current method). The reduced problem is the convex task of static optimization and can be solved with the use of many static optimization methods.

In the following subsections, we will present in turn the mathematical model of the task of medium flow (water), the reduction procedure and the optimization algorithm.

\subsection{Mathematical model of the water distribution network}

The main purpose of the water distribution network is to meet the needs of all recipients of the transported medium. To achieve this, the network must provide the recipients with the required amount of water of a given quality at the appropriate times [3, 4].

The problem formulated is generally the task of dynamic optimization with restrictions. The most commonly used quality optimization criterion is the price of electricity consumed by pumps located in pumping stations. The dynamics of the problem results, among others, from the presence of network tanks in the system. Restrictions result from the structure of the transport network and the parameters of particular elements.

An important element used in the construction of the mathematical model of the task is the random character of the recipients' needs. Decision variables in the problem are the number of pumps activated in pumping stations and the position of control valves located in various places on the network, most often at the pump station outlets.

The mathematical model of a water supply network should take into account, among others, the structure and topology of the network as well as non-linear characteristics of particular pipes and pumps in pumping stations. Let $l$ be the number of pump stations. We assume that pumps with identical characteristics are used in the pumping station. Each of these pumps consumes electricity in the amount specified by the general formula:

$$
P(y)=\alpha+\beta \cdot y \quad \alpha, \beta \geq 0
$$

where $y$ is the outflow from the pump. Formula (1) is called the pump power characteristic. If in the $i$-th pumping station works with and pumps yielding a total outflow $y_{i}$ then the power consumed by these pumps is determined by the formula:

$$
P_{i}\left(y_{i}, z_{i}\right)=\alpha_{i} \cdot z_{i}+\beta_{i} \quad i=1,2, \ldots, l
$$

The mathematical model of a single pipe is defined by Bernoulli's law:

$$
x_{i}=k_{i} \cdot y_{i}^{2} \cdot \operatorname{sgn}\left(y_{i}\right)+d_{i} \quad i=1,2, \ldots, n
$$

This is the relationship between the flow $y_{i}$ in the arc and the drop in water pressure at its ends $x_{i}$. The parameter $k_{i}$ is called the resistance of the pipe and $d_{i}$ is the difference in the height of the geodetic ends of this segment. A full description of the process of water distribution in the network is determined by Kirchhoff's I and II law:

- I Kirchhoff's law (Kirchhoff's current law)

$$
\boldsymbol{A} \cdot \boldsymbol{y}=\boldsymbol{p}
$$

- II Kirchhoff's law (Kirchhoff's loop rule)

$$
\boldsymbol{B} \cdot \boldsymbol{x}=\mathbf{0}
$$


In formula (4), $\boldsymbol{y} \in R^{n}$ is a vector constructed of flows in all arcs. $\mathbf{A}=\mathbf{A}_{\mathrm{mxn}}$ is an incident matrix. The parameter $m$ is the number of nodes of the network graph. The matrix $\mathbf{B}$ in the formula (5) is a loop matrix and $\boldsymbol{x} \in R^{n}$ is a vector constructed from pressure drops in individual $\operatorname{arcs} \boldsymbol{x}_{i}$.

\subsection{Description of an algorithm}

One of the most important problems in the water supply network is the determination of flows in all arcs of the network and pressures at all of its nodes. This requires the solution of a nonlinear system of algebraic equations (4) - (5).

Numerous techniques are known in the literature that allow to determine all flows and pressures in the network. We developed for these purposes our own method based on the principles of flow theory in networks.

The idea of the method is to transform the problem (4) - (5) into an equivalent static task of minimizing energy losses in all arcs of the network. This problem has the following form:

$$
f(\boldsymbol{y})=\sum_{i=1}^{n} f_{i}\left(y_{i}\right)
$$

subject to:

$$
A \cdot \boldsymbol{y}=\boldsymbol{p} .
$$

The objective function (6) is a sum of power losses in all arcs of the network. Each of its components is defined by the formula:

$$
f_{i}\left(y_{i}\right)=k_{i} \cdot y_{i}^{3} \cdot \operatorname{sgn}\left(y_{i}\right)+d_{i} \cdot y_{i} \quad i=1,2, \ldots, n
$$

and results from the Bernoulli's formula.

The algorithm of solving problem (4) - (5) developed by us, according to the idea of Newton's modified method, consists of three procedures: transformation to the problem of static optimization without restrictions, determination of the search direction for the reduced problem and the procedure of searching the minimum in the calculated direction.

Analyzes and computational tests performed have shown high efficiency of the developed procedure. The analytical complexity of the developed procedure for determining the minimum in the direction, measured number of multiplications, is equal to $n \cdot m$, where $n$ is the number of arcs and $m$ the number of nodes in the network under consideration.

\section{Summary and Future Plans}

The work presents a part of the results achieved by a group of employees of the Wroclaw University of Technology on the methods and algorithms created for the needs of control, optimization and design of non-linear transport networks. Over the last quarter of a century, there have also been works on gas distribution networks, IT networks, the task of identifying transport network parameters, etc.

Recent years, due to the specificity of the analyzed problems, resulted in works devoted to new optimization methods, based mainly on meta-heuristic idea. The obtained results and the results of numerical and computational tests performed confirm the effectiveness of the chosen approach and give hope for the application of the developed tools to many other complex optimization problems, such as raw material cutting tasks and many others.

Most of the developed methods and algorithms require the use of a computer with high computing power. WrUST aims at making an e-learning platform available to students. There would be teaching materials developed by teachers, mock tests as well as some space 
for students to exchange views and experience related to studying. The building is adapted for the needs of the disabled.

The teachers employed at the Complex of Academic Schools of WrUST have work experience with youth. Earlier many of them worked in the best junior secondary and secondary schools in Wroclaw.

In this area cloud computing is a useful tool in the modern Information Technology. IBM has invited Wroclaw University of Technology to join its two centers of Cloud excellence. One of them is an association of elite higher education institutions working in the Cloud Computing technologies called the IBM Cloud Academy. WrUST and IBM Poland plan to continue collaboration in the field of education, PHD programs and research. IBM plans to initiate similar programs with other institutes, faculties and data centers in Poland, as well as to integrate with local business communities in the process.

\section{References}

1. J. Kotowski, M. Ochla, J. Oko, APCASE 2014, Bali, Indonesia, Smart Cloud Orchestrator - the first implementation in the world at WrUST for supporting design processes in education at universities. To be published as a Chapter in the Springer book titled: "Theories, Methods and Applications of Computational Intelligence in Systems Engineering", (2014).

2. J. Kotowski, Computer aided systems theory - EUROCAST 2013: 14th International Conference, Las Palmas de Gran Canaria, Spain, Internships as an application of cloud computing solutions for education at universities, 10-15, (2013).

3. R. Klempous, J. Kotowski, JCAM, Some models for Water Distribution Systems, 26, 257-269, (1988).

4. R. Klempous, J. Kotowski, JCAM, Nonlinear transport network design, 35, 269-275, (1991). 